\title{
Meglitschia spp. (Myxozoa) Infecting the Gallbladder of Eugerres Brasilianus (Teleostei: Gerreidae) from the Atlantic Coast of Maceió (Brazil)
}

\begin{abstract}
Microscopic observations using light and transmission electron microscopes are used to describe a myxosporean belonging to the genus Meglitschia Kovaleva, 1988 (Phylum Myxozoa Grassé, 1970 found infecting the gallbladder of the marine teleost fish Eugerres brasilianus (Gerreidae) collected on the NE Atlantic coast of Brazil. Typical ellipsoidal furcate and arcuate $\cap$-shaped myxospores, identified as belonging to the genus Meglitschia were found infecting the gallbladder and described using light and transmission electron microscopy. Mature myxospores are composed of two symmetric equal-sized valves, with two equal-sized bifurcated caudal processes (tails). The myxospores observed free in the bile measures $24.6 \pm 0.8 \mu \mathrm{m}$ long, $8.4 \pm 0.5 \mu \mathrm{m}$ wide and $5.1 \pm 0.3 \mu \mathrm{m}$ thick and are composed of two symmetric equal-sized valves, up to $\sim 65 \mathrm{~nm}$ thick. Each valve possesses one opposed tapering appendage, $19.6 \pm 0.7 \mu \mathrm{m}$ long, oriented parallel towards the basal tip of the appendages and joined along a right suture line forming a thick strand. Two spherical and equal-sized polar capsules are located side by side in the same level in the central region of the myxospores body. Each polar capsule contains a polar filament coiled in 4-5 turns. Sporoplasm is binucleated and contains the nucleus just near the polar capsules surrounded by several globular sporoplasmosomes. The sporoplasm occupies the internal portion basal of the two caudal processes.
\end{abstract}

Keywords: Myxozoa; Marine; Fish infecting; Gallbladder; Ultrastructure

\begin{tabular}{|c|}
\hline Case Report \\
\hline Volume 5 Issue 3 - 2017 \\
\hline Themis Silva $^{1 *}$, Emerson Soares ${ }^{1}$, Sónia \\
\hline Rocha $^{2}$, Elton Santos ${ }^{3}$, Elsa Oliveira ${ }^{2}$, Graça \\
\hline Casall $^{1,4}$ and Carlos Azevedo $o^{2,3}$ \\
\hline $\begin{array}{l}{ }^{1} \text { Centre of Agricultural Sciences, Federal University of Alagoas, } \\
\text { Brazil }\end{array}$ \\
\hline $\begin{array}{l}{ }^{2} \text { Institute of Biomedical Sciences, University of Porto, Portugal } \\
{ }^{3} \text { Centre Interdisciplinary for Marine and Environmental }\end{array}$ \\
\hline $\begin{array}{l}\text { Research (CIIMAR/UP), University of Porto, Portugal } \\
{ }^{4} \text { Department of Sciences, University Institute of Heath North, } \\
\text { Portugal }\end{array}$ \\
\hline \multirow{3}{*}{$\begin{array}{l}\text { *Corresponding author: Themis Silva, Centre of } \\
\text { Agricultural Sciences, Federal University of Alagoas, Brazil, } \\
\text { Email: themisjdasilva@gmail.com }\end{array}$} \\
\hline \\
\hline \\
\hline Received: May 24, 2017 | Published: July 17, 2 \\
\hline
\end{tabular}

Abbreviations: PFc: Polar Filament Coils; GB: Gallbladder; DIC: Differential Interference Contrast; TEM: Transmission Electron Microscopy; PC: Polar Capsules

\section{Introduction}

The Myxozoa Grassé, 1970 infecting fish is an important pathogenic group having a worldwide distribution. Among these parasites the monotypic genus Meglitschia Kovaleva, 1988 has been reported infecting the gallbladder $(\mathrm{Gb})$ of a marine fish from the New Zealand fauna [1,2] and a freshwater fish from Brazil, collected in a tributary of Amazon River [3]. The teleostean host (Eugerres brasilianus) a commercially important fish of the family Gerreidae, common in the NE Atlantic coast of Brazil, was infected Gallbladder by myxospores of the genus Meglitschia. This genus is a small taxonomic group within the class Myxosporea that contains only two named species, M. insolita [1] (formerly described as Ceratomyxa sp.) [2] from New Zealand and M. mylei [3] from Brazil. These two species were described on the base of a schematic drawing [1] and based in light and transmission electron microscopies [3]. The present study provides light and ultrastructural for the description of Meglitschia sp., a myxosporean parasite infecting the Gallbladder of a marine fish on the Brazilian northern Atlantic coast. This genus, despite being similar to Ceratomyxa spp. with which it shares some morphologic aspects, differs in the typically arcuate $\cap$-shaped of their myxospores.

\section{Case Description}

Forty specimens (27 females and 13 males) of the marine fish Gallbladder of the teleostean fish Eugerres brasilianus (Teleostei: Gerreidae) (Brazilian common name "Carapeba" or "Mojarra") were collected during October and November 2015, from the Atlantic coast $\left(09^{\circ} 29^{\prime} \mathrm{S} / 35^{\circ} 34^{\prime} \mathrm{W}\right)$ near the city of Maceió (State of Alagoas), Brazil. Specimens measured $30-40 \mathrm{~cm}$ in length and weighed 600-700 g. and the respective sex register. A parasitological survey was conducted on several organs and tissues. Parasitized samples from the infected host specimens were examined and photographed using the light microscope LEICA Leitz DMBRE, equipped with differential interference contrast (DIC) optics and the digital camera LEICA DFC480 (LEICA Microsystems, Wetzlar, Germany). Measurements were taken using the software LAS V4.3 (LEICA Microsystems).

For transmission electron microscopy (TEM) were used living and refrigerated samples to obtained myxospores morphologically identified as belonging to the genus Meglitschia and fixed in 5\% glutaraldehyde buffered in $0.2 \mathrm{M}$ sodium cacodylate $(\mathrm{pH} 7.4)$ for 20-24h, washed overnight in the same buffer, and postfixed in 
$2 \% \mathrm{OsO}_{4}$ also buffered with $0.2 \mathrm{M}$ sodium cacodylate ( $\mathrm{pH} 7.4$ ) for $3-4 \mathrm{~h}$. All these steps were performed at $4^{\circ} \mathrm{C}$. The samples were then dehydrated in an ascending graded series of ethanol, followed by embedding using a series of oxide propylene and Epon mixtures, ending in EPON. Semithin sections were stained with methylene blue Azur II. Ultrathin sections were double-contrasted with uranyl acetate and lead citrate, and then examined and photographed using a JEOL 100CXII TEM (JEOL Optical, Tokyo, Japan), operating at $60 \mathrm{kV}$. No ultrastructural differences were found on myxospores obtained from the fixation of living samples and myxospores obtained from samples death some hours before (5-10h) and conserved in glace before the chemical fixation with glutaraldehyde. The observation in aquarium of the living fish does not present altered behavior. The typical morphology of the myxospores suggests that the present microparasite belongs to the genus Meglitschia, according the previous descriptions [1-4].

\section{Gallbladder and bile}

The infected Gallbladder appeared sometimes swelled and having a light brownish color when contains numerous myxospores floating in the bile. Microscopic observations revealing myxospores parasitizing the Gallbladder of some specimens ( $17 \%$ infected). Numerous myxospores floating in the light brownish bile and, among them, several plasmodia containing different developmental stages were observed (Figure 1). No other organ seemed infected with same type of myxospores or plasmodia.

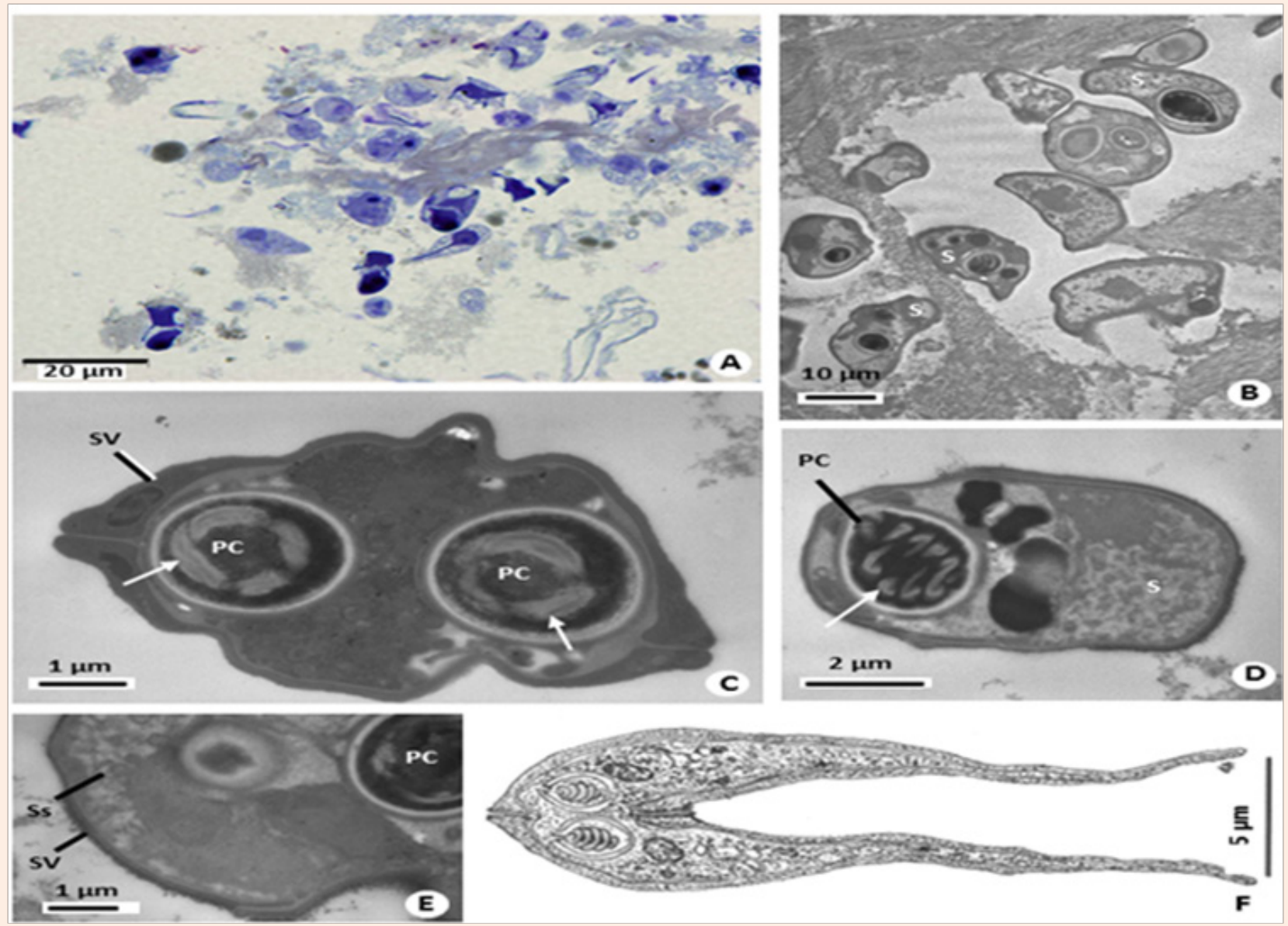

Figure 1: A. Semithin section showing several myxospores in the bile. B. TEM micrograph showing some Myxospores(S) sectioned at different levels. C. Transverse section showing the two polar capsules (PC) located side by side, the polar filament (arrows), and the valves comprising the wall (SW). D. Longitudinal ultrathin section of a polar capsule (PC) displaying the organization of the polar filament (arrow), and the sporoplasm cell (SC). E. Ultrathin section of the periphery of a myxospore showing the valves (SV), sporoplasmosomes (Ss), and part of the polar capsule (PC). F. Schematic drawing of a myxospore depicting its ultrastructural aspects.

\section{Mature myxospores}

The bile was collected and the isolated myxospores, as well small fragments of the Gb wall prepared for LM and TEM. Observed in LM and TEM, myxospores strongly furcate and arcuate $\cap$-shaped, averaging $\sim 24 \mu \mathrm{m}$ in length, $8-9 \mu \mathrm{m}$ in width and $\sim 5 \mu \mathrm{m}$ in thickness. Wall composed of two equal-sized valves (Figures $1 \mathrm{~A}-1 \mathrm{E}$ ), each possessing one opposed tapering appendage, $\sim 20 \mu \mathrm{m}$ long, oriented parallel towards the basal tip of the appendages and joined along a straight suture line forming a thick strand. The strand goes around the central part of the myxospore, which in turn surrounds two equal and symmetric 
spherical polar capsules (PC), 2-3 $\mu \mathrm{m}$ in diameter, located at the same level. Each PC contained a polar filament forming 4-5 coils. The binucleate sporoplasm was irregular in shape, contained several sporoplasmosomes and fully occupied the space of the two caudal appendages (Figures $1 \mathrm{C}-1 \mathrm{E})$. The nuclei $(\sim 1.9 \mu \mathrm{m}$ in diameter) were located near the PC. A schematic drawing of the myxospore organization, obtained from the serial longitudinal ultrathin sections, is shown in the Figure $1 \mathrm{~F}$.

\section{Discussion}

The morphological and ultrastructural aspects of the myxospores showing a furcate and arcuate $\cap$-shaped organization show that these myxospores are similar with those previously described belonging to the genus Meglitschia [1-4]. Based on the arc shape of the myxospore with two tapering caudal appendages oriented to the basis of spores, on the number and position of the $\mathrm{PC}$ and of the polar filament coils (PFc) and arrangements, the morphology of the myxospores suggested that this parasite belongs to genus Meglitschia. This myxoparasite was the first of the reported genus infecting $E$. brasilianus, where there is only one species of this genus (Meglitschia mylei) described parasitizing fish in Brazil [3]. Based on the some morphological differences in terms of size, shape and the ultrastructural details of the myxospores and PC and PFc, when compared with those myxospores of the two species previously described, we observe that the present described isolated shows several morphologic differences to the two published species. However, the lack of the phylogenetic data on these two species does not permit the comparison with our phylogenetic results.

\section{Conclusion}

Upon comparison to other know Meglitschia sp., the morphometric and ultrastructural data obtained for the parasite described here, suggest that it is, possibly, to be a new member of this genus. Nonetheless, species identification is dependent of the acquisition of further information, namely at molecular level.

\section{Acknowledgement}

This work was partially supported by Engo. A. Almeida Foundation (Porto, Portugal) and Project SSPP \#0067 of King Saud University. The work was partially supported by FAPEALFoundation of Research of Alagoas State, Brazil.

\section{References}

1. Meglitsch PA (1960) Some coelozoic Myxosporidia from New Zealand fishes. I. General and family Ceratomyxidae. Trans R Soc N Z 88(2): 265-365.

2. Kovaleva AA (1988) Suborder Sphaeromyxina (Myxosporea, Bivalvulida) its structure and place in myxosporidian classification. Zool Zh 67: 1616-1620.

3. Azevedo C, Ribeiro M, Clemente SCS, Casal G, Lopes L, et al. (2011) Light and ultrastructural description of Meglitschia mylei n. sp. (Myxozoa) from Myleus rubripinnis (Teleostei: Serrasalmidae) in the Amazon River System. J Eukaryot Microbiol 58(6): 525-528.

4. Lom J, Dyková I (2006) Myxozoan genera: definition and notes on taxonomy, life-cycle terminology and pathogenic species. Folia Parasitol (Praha) 53(1): 1-36. 